Journal of Computer Science 5 (8): 557-563, 2009

ISSN 1549-3636

(C) 2009 Science Publications

\title{
Contour Following Task with Dual Sensor Logic Algorithm for Adept Selective Compliant Assembly Robot Arm Robot
}

\author{
${ }^{1}$ Anton Satria Prabuwono, ${ }^{2}$ Samsi Md. Said, ${ }^{1}$ Riza Sulaiman and ${ }^{1}$ Elankovan Sundararajan \\ ${ }^{1}$ Faculty of Information Science and Technology, \\ University Kebangsaan Malaysia, 43600 UKM Bangi, Selangor DE, Malaysia \\ ${ }^{2}$ Faculty of Electrical and Automation Engineering Technology, \\ TATi University College, Teluk Kalong, 24000 Kemaman, Terengganu, Malaysia
}

\begin{abstract}
Problem statement: The capability of performing autonomously a contour following task of a shape object is required for an industrial robot manipulator. These tasks include welding, sealing, grinding, painting and many more. These applications are very tedious and time consuming if done by manual contour following system. Approach: This study proposed the development of dual sensor logic algorithm for contour following task in order to automate the manual programming process. Results: The outcome of this study was to make industrial robot capable of doing self learning to follow the contour with minimal human involvement. The algorithm had been developed and tested using Adept Selective Compliant Assembly Robot Arm (SCARA) robot. Conclusion: The result showed its possibility could be used as automated contour following task for real industrial robot.
\end{abstract}

Key words: Dual sensor logic, contour following, adept SCARA robot

\section{INTRODUCTION}

The current trend towards industrial robotics requires the implementation of simple programming techniques. Programming by demonstration has emerged as one of the most promising solutions for effective programming of robot tasks ${ }^{[1,2]}$. Contour following is one of basic task in industrial robot manipulation. In the task, the robot is holding a tool to follow the contour of an object whose shape and pose are often unknown ${ }^{[3]}$. These applications include part polishing, inspection, sealing, painting, cleaning, modeling, etc. During the tracking, the tool is constrained on the surface to maintain contact force while moving along some tangential direction. A lot of efforts have been done in automating and enhancing teaching process of the robot applications as described in $^{[4-6]}$.

In order to use robotics for such application, two sequences of step need to be considered i.e., the programming phase and the playback phase. In the programming phase, teaching a group of points is required while for playback phase, the robot Tool Centre Point (TCP) will follow the taught points recorded previously. This programming phase especially for contour following application such as in arc welding, sealing and painting application is quite tedious and time consuming ${ }^{[7]}$. For example, in order to follow an arc, the robot programmer needs to manually use teaching box or teaching pendant to jog the robot TCP to three points that enclosed an $\operatorname{arc}^{[8]}$. Furthermore, the current Flexible Manufacturing System (FMS) concept requires different kind of parts variations for one production run. This means that a great number of robot programming is required to cater parts variations and uncertainties per production run compared to the old days of batch or mass production concept.

In this study, we propose the implementation of dual sensor logic algorithm for contour following task in real industrial robot. The algorithm has been developed and tested using Adept SCARA robot. By employing a simple programming and solution, the robot demonstrates intelligent behavior to follow the contour of object shape.

Basic concept of contour following task: Figure 1 shows a diagram of contour following task for the SCARA robot ${ }^{[9]}$. Frame (0) refers to the robot base, while task frame (T) has its origin on the robot endeffector with its $\mathrm{n}$ and $\mathrm{t}$ axes that are directed respectively along the normal and tangential direction of contour of the piece, whose geometry is assumed to

Corresponding Author: Anton Satria Prabuwono, Faculty of Information Science and Technology, University Kebangsaan Malaysia, 43600 UKM Bangi, Selangor DE, Malaysia 
be unknown. The $\varphi$ is the angle between $\mathrm{n}$ axis and $\mathrm{x}$ axis of frame (0). Let:

$$
\mathrm{Q}=[\mathrm{q} 1, \mathrm{q} 2]^{\mathrm{T}}
$$

Be the vector of the joints position and $\mathrm{Q}$ its first time derivative. Since a suitable belt transmission keeps the end-effector with constant orientation with respect to the absolute frame, force measurements are directly available in frame (0). Let:

$$
F_{(0)}=\left[f_{x}, f_{y}\right]^{T}
$$

and

$$
\mathrm{F}_{(\mathrm{T})}=\left[\mathrm{f}_{\mathrm{t}}, \mathrm{f}_{\mathrm{n}}\right]^{\mathrm{T}}
$$

Be the vector of contact force in frame (0) and (T) respectively. They are related to each other by the equation:

$$
\mathrm{F}_{(0)}=\mathrm{M}_{\mathrm{T} 0}(\vartheta) \mathrm{F}_{(\mathrm{T})}
$$

Denoting with $\mathrm{M}_{\mathrm{ij}}$ the rotation matrix converting vectors from frame $j$ to $i$. Vector $V_{(T)}=\left[v_{t}, v_{n}\right]^{T}$ representing Cartesian velocity in frame $(\mathrm{T})$ can be obtained from:

$$
\mathrm{V}_{(\mathrm{T})}=\mathrm{M}_{\mathrm{T} 0} \mathrm{~V}_{(0)}=\mathrm{M}_{\mathrm{T} 0} \mathrm{~J}(\mathrm{Q}) \dot{\mathrm{Q}}
$$

Where:

$$
\mathrm{M}_{\mathrm{T} 0}=\left[\begin{array}{cc}
-\sin \vartheta & \cos \vartheta \\
\cos \vartheta & \sin \vartheta
\end{array}\right]
$$

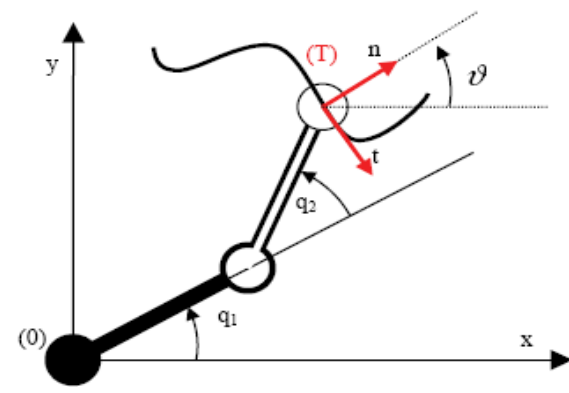

Fig. 1: Diagram of contour following task for SCARA robot and $\mathbf{J}(\mathrm{Q})$ is the Jacobian matrix for which:

$$
\mathrm{V}_{(0)}=\mathrm{JQ}
$$

Aim of the contour following task is to control the normal force and tangential velocity of the robot endeffector along the $\mathrm{n}$ and $\mathrm{t}$ directions of the task frame (T). These directions can be easily detected assuming negligible the friction force in the tangential direction (this is achieved by providing a ball bearing at the probe) by on-line estimating the angle $\vartheta$ as:

$\vartheta=a \tan 2\left(f_{x}, f_{y}\right)=\arctan \left(\frac{f_{x}}{f_{y}}\right) \pm \pi$

In industrial application like grinding the tangential friction force cannot be neglected. In this case, the contact direction estimation (Eq. 8) can be suitable compensated using cutting force models ${ }^{[10]}$ or measuring the torque of the milling spindle ${ }^{[11]}$.

\section{MATERIALS AND METHODS}

Smart tool holder and sensors: Smart tool is designed to hold two digital proximity sensors of different sensing distance as shown in Fig. 2-4.

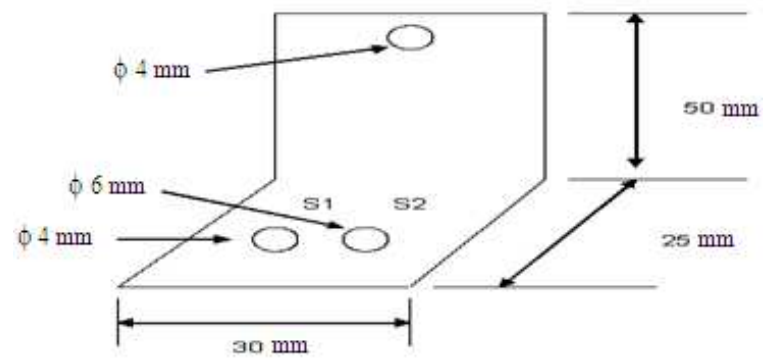

Fig. 2: Dimension of smart tool holder

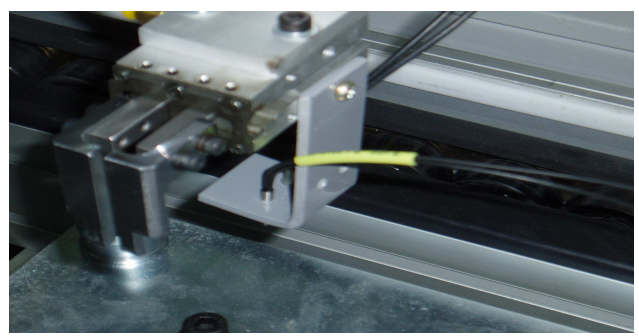

Fig. 3: Tool holder attached to Adept SCARA robot TCP 


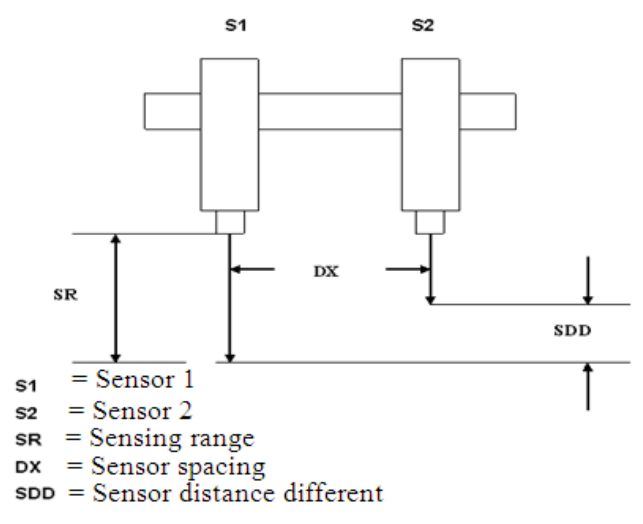

Fig. 4: Tool holder attached to Adept SCARA robot TCP

This Sensing Distance Difference (SDD) is measured by finding the downward vertical variable difference and is adjusted to be as low as $1 \mathrm{~mm}$ to as high as $5 \mathrm{~mm}$. Sensing Range (SR) is adjusted of about $15 \mathrm{~mm}$ for safety reason to avoid collision to contour. In order to accommodate mechanical design of sensor, the sensor spacing (DX) is fixed to $10 \mathrm{~mm}$. The contour slope sensitivity index is a ratio of SDD over DX. The lesser the index value the more sensitive the contour slope to be sensed.

The initial job has been defined in the teaching position point of view. The purpose of this initial job is to demonstrate the working principle of the general idea and then in the second part, several simpler solutions of using one sensor algorithm concept are developed. In future, further research needs to be taken in order to find the optimal combination of these parameters versus the contour variable slope.

Sensing strategy: The Sensor number 1 and 2 will detect the current contour gradient whether it is positive climbing, negative descending or just horizontal flat. The contour gradient based on the sensors 1 and 2 logic conditions are described as follow:

- If both sensors were switched on, then TCP move up by SDD $\mathrm{mm}$ and move horizontally by SDD mm

- If both sensors were switched off, then TCP move down by SDD $\mathrm{mm}$ and move horizontally by SDD mm

- If sensor one was on and sensor two was off, move horizontally by SDD $\mathrm{mm}$ or until condition a, or condition $\mathrm{b}$ was reached (whichever comes earlier)

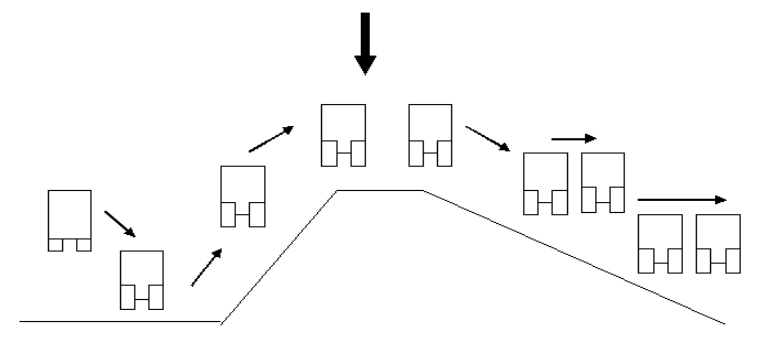

Fig. 5: Tool movement of sensors

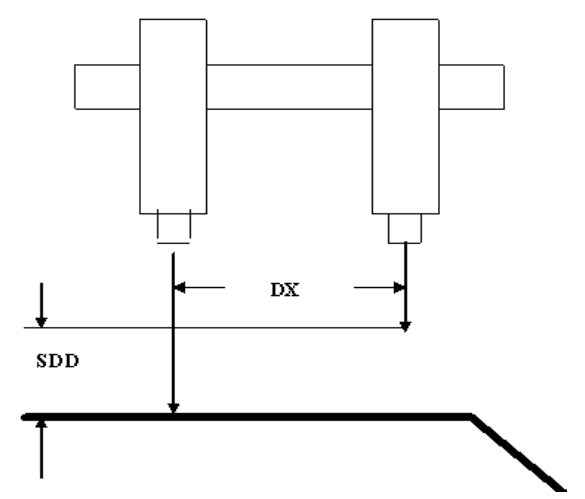

Fig. 6: Sensors in flat horizontal operation

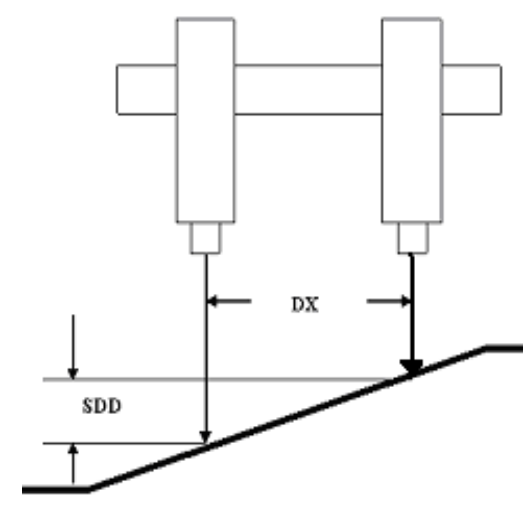

Fig. 7: Sensors in climbing operation

Table 1: Movement logic for sensors

\begin{tabular}{lll}
\hline S1 & S2 & Operation \\
\hline 0 & 0 & Descend \\
1 & 0 & Horizontal \\
flat & & \\
1 & 1 & Ascend \\
\hline
\end{tabular}

Movement logic of sensors is shown in Table 1. Tool movements and operations are shown in Fig. 5-8.

With the next incremental motion of point $\mathrm{P}(\mathrm{i}+1)$ in the $\mathrm{X}-\mathrm{Z}$ plane. Cartesian coordinate of point $\mathrm{P}(\mathrm{i})$ is actually a transformation from individual joint axes. 


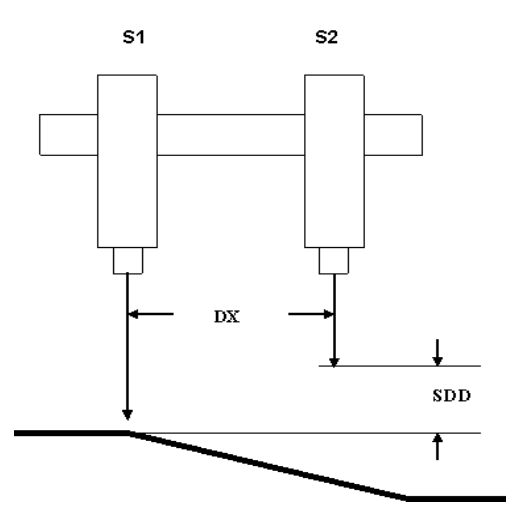

Fig. 8: Sensors in descending operation

Algorithm: The programming will take advantage of the current information of both Sensor 1 and 2. If both sensors' reading are on that means at current location the contour slope is positive climbing. If only sensor 1 reading is on that means TCP on a flat horizontal shape. If both sensors' reading are off then the TCP is on descending mode. The next motion of $\mathrm{P}(\mathrm{i}+1)$ is being commanded using current data and be tested using same rules. The program starts with user definition of contour length as configured previously.

The algorithm will find the slope in $\mathrm{X}-\mathrm{Y}$ plane and total distance diagonally and $\mathrm{x}$ and $\mathrm{y}$ vector distance. If the starting point is greater than sensor 1 Sensing Range (SR), both sensors input to the robot controller is in 0 volt (off) and condition descending is applied. Robot will incrementally move downward until the contour will within sensor 1 Sensing Range (SR). At this point the sensor 1 input value is 24 volt (on) while sensor 2 value is zero volt (off). This indicates flat condition and the robot will move horizontally until it senses the climbing positive slope contour where both sensors 1 and 2 reading's are on. At this stage the condition climbing is applied and robot will climb. The transition of variable slope changing from positive slope to flat and negative slope will cause the program to implement "noise motion".

In climbing mode, any variable slope transition vertex will cause "flat noise motion" while in descending mode multiple "flat noise motion" staircase deviation happen due to both sensors are off in descending logic. Noise study and optimization were carried out in this research especially in descending mode. These phenomena will be improved in future research. Fig. 9 shows dual sensor logic program flowchart.

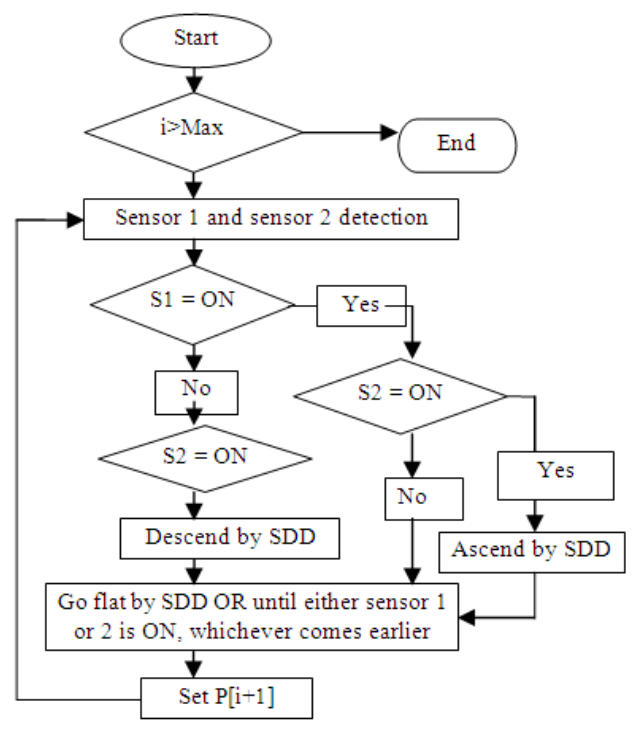

Fig. 9: Flowchart of dual sensor logic program

Drive transform modeling: Smart sensor feedback and programming algorithm will guide the TCP to approximate the curve with a straight line segments that knot from points to points in three dimensional Cartesian X-Y-Z plane. The measured knot points and segment slope at any points will be stored in the database and will be used repeatedly in robot part program playback mode. The objectives of automating tedious and time consuming contour tracking programming process will be achieved. Further explain the incremental position of $\delta_{\mathrm{x}}, \delta_{\mathrm{Y}}$ and $\delta_{\mathrm{Z}}$, of general incremental drive transform described in Eq. 11. For start, in order to simplify the mathematical formulation, four degree of freedom Adept SCARA industrial robot is used.

In future research, a six degree of freedom robot can be used to test the robustness and applicability of the algorithms. Utilizing drive transform equation for four degree of freedom SCARA robot will simplify a lot of things ${ }^{[12,13]}$. For example, only one yaw orientation angle exist. Then, the chord segment relative path transformation drive transform is being decomposed only into one rotation matrix to orientate tool about $\mathrm{Z}$ axis and one straight line translation matrix also along tool axis. In order to achieve the motion between two consecutive Cartesian knot points, the derivation of segment drive transform is very useful since motion from $i-i+1$ is related to drive transform as:

$\mathrm{T}_{4}(\mathrm{i}+1)=\mathrm{C}_{\text {workobject }} \mathrm{P}_{\mathrm{i}} \mathrm{D}(\mathrm{i}+1)\left({ }^{\text {tool }} \mathrm{T}_{\mathrm{i}+1}\right)^{-1}$ 
$\mathrm{T}_{4}(\mathrm{i}+1)$ is the transformation stored to the database and contain both tool position and orientation at any points which also becomes input to the inverse kinematics routine in order to get local coordinate of individual robot joint angles (another joint level cubic polynomial trajectory planning or differential Jacobian method which is not discussed here). After some mathematical operation, the position of consecutive knot points at beginning from $i$ to end of segment $i+1$ is a function of drive transform as:

$\mathrm{P}_{\mathrm{i}+1}=\mathrm{P}_{\mathrm{i}} \mathrm{D}(\mathrm{i}+1)$

The general transformation matrices drive transform that summarizes all these can be represented as:

$$
\mathrm{D}(\mathrm{i}+1)=\left[\begin{array}{cccc}
\mathrm{C}(\theta) & 0 & \mathrm{~S}(\theta) & \delta_{\mathrm{x}} \\
0 & 1 & 0 & \delta_{\mathrm{y}} \\
-\mathrm{S}(\theta) & 0 & \mathrm{C}(\theta) & \delta_{\mathrm{z}} \\
0 & 0 & 1 & 1
\end{array}\right]
$$

The yaw orientation angle $\theta$ is actually an input from gross motion task planning that was discussed previously. The detail of incremental position in Cartesian X-Y-Z three dimensional plane $\delta_{\mathrm{x}}, \delta_{\mathrm{y}}$ and $\delta_{\mathrm{z}}$ will be explained in several individual alternate algorithm developments in the results just to automate this differential relative motion. Incremental drive transform that will describe the final position at any point $\mathrm{N}$ is generated as:

$\mathrm{P}_{\mathrm{N}}=\prod_{\mathrm{i}=1}^{\mathrm{N}-1} \mathrm{P}_{\mathrm{i}} \mathrm{D}(\mathrm{i}+1)$

The related transformation at any point $\mathrm{N}$ which became input to inverse kinematics routine for joints space trajectory planning as follow:

$\left.\mathrm{T}_{4}(\mathrm{~N})=\prod_{\mathrm{i}=1}^{\mathrm{N}} \mathrm{C}_{\text {worobject }} \mathrm{P}_{\mathrm{i}} \mathrm{D}(\mathrm{i}+1){ }^{{ }^{\text {tol }}} \mathrm{T}_{\mathrm{i}+1}\right)^{-1}$

Experiment: The V+ programs from those algorithms will be written and will be tested using an Adept SCARA robot. The contours traced by robot TCP on the semicircle object which has radius value of $40 \mathrm{~mm}$ along the $\mathrm{X}$ axis are plotted according to the algorithm used. All actual contour coordinate traced at any point will be compared to the known geometry contour shape equation in order to find tracking error at any point $i$. The error between actual coordinated traced by robot TCP and known coordinate value of semicircle geometry at any time $\mathrm{i}$ is:

$\varepsilon_{\mathrm{i}}=\mathrm{w}_{\mathrm{i}}-\mathrm{z}_{\mathrm{i}}$

Where:

$\mathrm{W}_{\mathrm{i}}=$ Actual geometry

$\mathrm{z}_{\mathrm{i}}=$ The contour traced

Two graphs are plotted for individual algorithm such as the actual contour traced versus semicircle geometry, tracking error versus contour geometry (all along $\mathrm{X}$ axis). The mean of tracking error will be used to measure the performance index of proposed algorithm for all point $\mathrm{N}$ captured as follow:

$\bar{\varepsilon}=\frac{1}{\mathrm{n}} \sum_{\mathrm{i}=1}^{\mathrm{N}} \varepsilon_{\mathrm{i}}^{2}$

Another criterion to measure the error distribution is by employing standard deviation as follow:

$\mathrm{S}=\left[\frac{1}{\mathrm{n}} \sum\left(\varepsilon_{\mathrm{i}}-\varepsilon_{\mathrm{i}}\right)^{-2}\right]^{\frac{1}{2}}$

Semicircle shape was chosen because it provides an ideal test bed because it contains all ranges of slope gradient that are available in real world. It exhibit infinity value at the very beginning point and progressing down with a finite very high positive slope. The slope decreasing into zero value in the middle of the contour and finally reaches very high negative slope at the other end along the $\mathrm{X}$ axis. At the very end of the semicircle contour the infinity slope reappear again. These phenomena cause high reading of Cartesian vector $\mathrm{Z}$ for any minute vector $\mathrm{X}$ displacement value. These infinity region problems will be avoided by introducing a safety margin ranging from $0.1-2.5 \mathrm{~mm}$ at the both ends of the semicircle geometry. It is anticipated that the tracking error value will be quite high in certain slope region of contour gradient. This limitation will be discovered and discussed again in the result. Figure 10 shows final line position teaching in the experiments. 


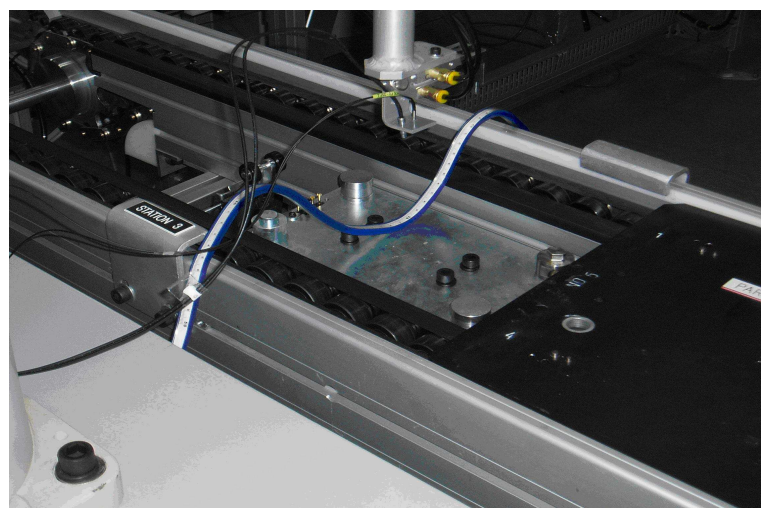

Fig. 10: Final line position teaching

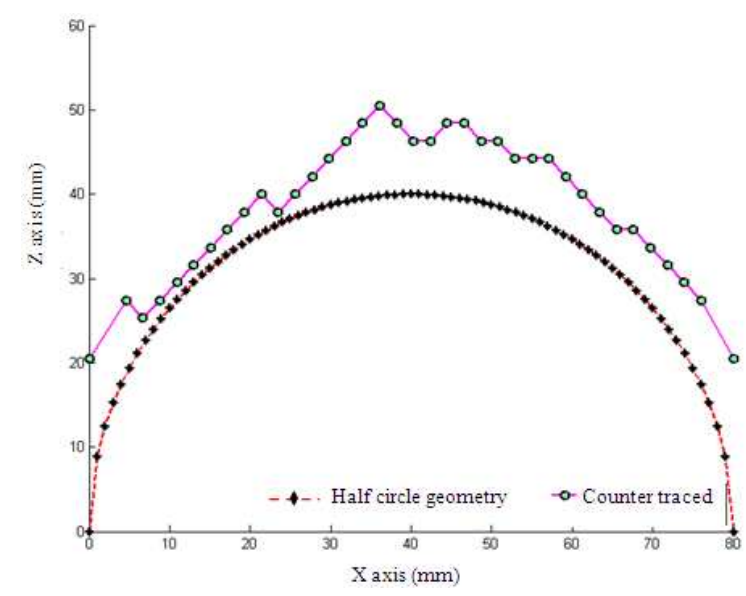

Fig. 11: Contour traced along half circle geometry with dual sensor

\section{RESULTS}

The dual sensor logic algorithm has been developed and tested using Adept SCARA robot in the real environment. The actual contour traced and the tracking error along contour, matching the semicircle geometry of radius $40 \mathrm{~mm}$ is plotted in Fig. 11 and 12 .

The enlargement of mean of tracking error with the value of $13.7015 \mathrm{~mm}$ and the standard deviation of tracking error with the value of $4.1247 \mathrm{~mm}$ respectively. The safety margin of $0.1-1 \mathrm{~mm}$ is allowed at the beginning and near to the end of semicircle object in order to avoid measuring the very high slope at those regions. The sensor difference between Sensor 1 and 2 (SDD) value is about 2.21 $\mathrm{mm}$ giving a bit coarse measurement points spaced apart at every $2.21 \mathrm{~mm}$. The total sample of 35 points was collected over $80 \mathrm{~mm}$ horizontal measuring distance.

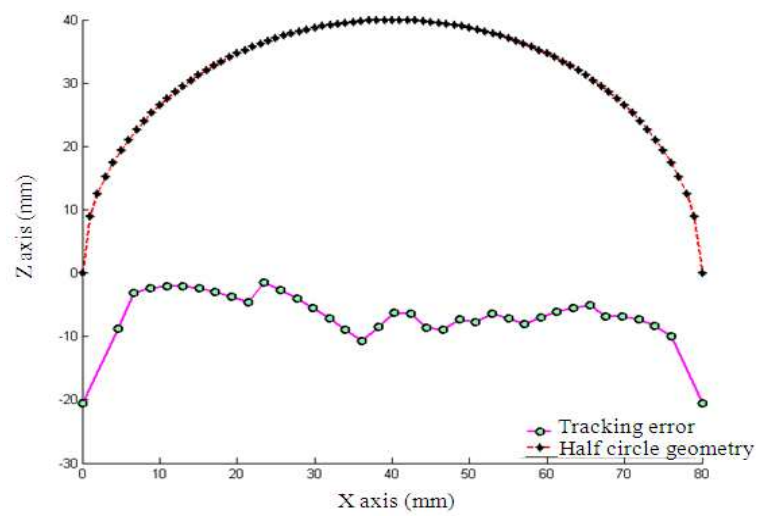

Fig. 12: Tracking error along half circle geometry with dual sensor

\section{DISCUSSION}

In this study the autonomous contour following task with dual sensor logic algorithm has been developed for Adept SCARA robot. A prototype of smart tool integrated with sensor has been designed. It can be attached and reattached into robot gripper and interfaced through $\mathrm{I} / \mathrm{O}$ pins of Adept robot controller for automated robot teaching operation. The algorithm developed was tested on a semicircle object of $40 \mathrm{~mm}$ radius. The semicircle object was selected because it exhibits the stringent test bed which provides the changing gradient gradually from steepest positive slope into zero slope of flat curve in the middle and finally to steepest negative slope. This test exhibits the performance of algorithm used which proves its possibility to be applied in the real world application.

\section{CONCLUSION}

This research demonstrates the autonomous contour following points recording for the robot teaching process can be successfully achieved. The programming to implement those algorithms is also developed. The findings allow simple automation of robot teaching process for several industrial applications such as robotic spray painting application, robotic arc welding application, robotic windshield glass sealing application, coordinate measuring machine, robot capturing three dimensional contour surfaces. For the future, automatic curve radius determination between straight line segments can be improved by integrating vision system for the automation of top view (X-Y coordinate) edge finding and path planning. The integration of vision system with 
the present study will improve the automation level of the project from two to three dimensional capabilities.

\section{ACKNOWLEDGMENT}

The researchers would like to thank Faculty of Information Science and Technology, University Kebangsaan Malaysia for providing facilities and financial support.

\section{REFERENCES}

1. Ikeuchi, K. and T. Suehiro, 1994. Towards an assembly plan from observation, Part I: Task recognition with polyhedral objects. IEEE Trans. Robot. Automat., 3: 368-385. DOI: 10.1109/70.294211

2. Zollner, R., O. Rogalla, R. Dillmann and M. Zollner, 2002. Understanding Users Intention: Programming Fine Manipulation Tasks by Demonstration. Proceeding of the IEEE/RSJ International Conference on Intelligent Robots and Systems, (IRS'02), IEEE Xplore Press, USA., pp: 1114-1119. DOI: 10.1109/IRDS.2002.1043880

3. Mi, L. and Y.B. Jia, 2004. High precision contour tracking with joystick sensor. Proceeding of the IEEE/RSJ International Conference on Intelligent Robots and Systems, Sept. 28-Oct. 2, IEEE Xplore Press, USA., pp: 804-809. DOI: 10.1109/IROS.2004.1389451

4. Adolfo, B., C.A.A. Sadek and A.D. Leszek, 2001. Predictive sensor guided robotic manipulators in automated welding cells. J. Mater. Process. Technol., 9: 13-19. DOI: 10.1016/S09240136(00)00771-8

5. Yuehong, Y., H. Hui and X. Yanchun, 2004. Active tracking of unknown surface using force sensing and control technique for robot. Sensors Actuators: A Phys., 112: 313-319. DOI: 10.1016/j.sna.2004.01.044

6. Gopalakrishnan, B., S. Tirunellayi and R. Todkar, 2004. Design and development of an autonomous mobile smart vehicle: A mechatronics application. Mechatronics, 14: 491-514. DOI: 10.1016/j.mechatronics.2003.10.003
7. Prabuwono, A.S. and M.S. Samsi, 2007. Development of adapting gradient method for contour tracking in industrial robot application. Proceeding of the 10th IASTED International Conference on Intelligent Systems and Control, (ISC'07), Hawaii, USA., pp: 592-068. http://www.actapress.com/PaperInfo.aspx?PaperID $=32176 \&$ reason $=500$

8. Prabuwono, A.S., M.A. Burhanuddin and M.S. Samsi, 2008. Autonomous contour tracking using staircase method for industrial robot. Proceeding of the 10th IEEE International Conference on Control, Automation, Robotics and Vision, Dec. 17-20, IEEE Xplore Press, Hanoi, pp: 2272-2276. DOI: 10.1109/ICARCV.2008.4795886

9. Ziliani, G., A. Visioli and G. Legnani, 2006. Gain scheduling for hybrid force/velocity control in contour tracking task. Int. J. Adv. Robot. Syst., 4: 367-374.

http://www.intechweb.org/downloadpdf.php?id=42 03\&PHPSESSID $=\mathrm{a} 1 \mathrm{vl} 23 \mathrm{bu} 5 \mathrm{c} 1 \mathrm{k} 0 \mathrm{dl} 1$ povb22gdl6

10. Duelen, G., H. Munch, D. Surdilovic and J. Timm, 1992. Automated force control schemes for robotic deburring: development and experimental evaluation. Proceeding of the International Conference on Industrial Electronics, Control, Instrumentation and Automation, Nov. 9-13, IEEE Xplore Press, San Diego, CA., USA., pp: 912-917. DOI: 10.1109/IECON.1992.254483

11. Ziliani, G., G. Legnani and A. Visioli, 2005. A mechatronic design for robotic deburring. Proceedings of the IEEE International Symposium on Industrial Electronics, June 20-23, IEEE Xplore Press, USA., pp: 1575-1580. http://ieeexplore.ieee.org/xpl/freeabs_all.jsp?arnum ber $=1529167$

12. Paul, R., 1979. Manipulator Cartesian path control. IEEE Trans. Syst., Man, Cybernet., 9: 702711. DOI: 10.1109/TSMC.1979.4310109

13. Paul, R.P.C., 1972. Modeling, trajectory calculation and servoing of a computer controlled arm. Ph.D. Dissertation, Stanford University, CA., USA. http://portal.acm.org/citation.cfm?id=906014 